\title{
TEMPORAL DISCOUNTING FOR MULTIDIMENSIONAL ECONOMIC AGENTS
}

\author{
FLORENTIN BOTA
}

\begin{abstract}
Individuals frequently place a higher value on money and goods today than they would in the future. This is known as temporal or time discounting, and most economic models include discount functions to represent such utility over time.

In this paper we evaluated traditional models with experimental data from the scientific literature and constructed our own samples for comparison. In addition, we evaluated the prediction accuracy of the models and proposed new hybrid solutions. Our investigation aims to contribute to a better understanding of human nature in complex processes.
\end{abstract}

\section{INTRODUCTION}

We propose a new unified computational model that represents the human decision-making process in complex systems, such as economy. The model is created using a bottom-up, data-driven approach[5] and will provide an effective tool for developing realistic Multidimensional Economic Agents (MEA). This paper focuses on the rational part of the model, where we analyze the standard paradigm, specifically the time-preference or temporal discounting phenomenon observed in economy.

Intertemporal choice is an elegant and simple economic theory introduced by John Ray in 1834 and formulated by Irving Fisher (1930) [9, 25], who created a model that includes an individual's impatience, contrary to Keynes (1936) [13], who emphasized on current income in relation with consumption. In this model the consumers make time-based decisions to maximize their lifetime satisfaction.

Figure 1 is a representation of intertemporal choice of the consumer subject to the utility preferences and the budget constraint. The indifference

Received by the editors: 3 April 2021.

2010 Mathematics Subject Classification. 68Q19, 91B44.

1998 CR Categories and Descriptors. J.4 [Computer Applications]: Social and Behavioral Sciences - Economics; I.6.4 [Computing Methodologies]: Simulation and Modeling - Model Validation and Analysis .

Key words and phrases. economics, temporal discounting, intertemporal decisions. 


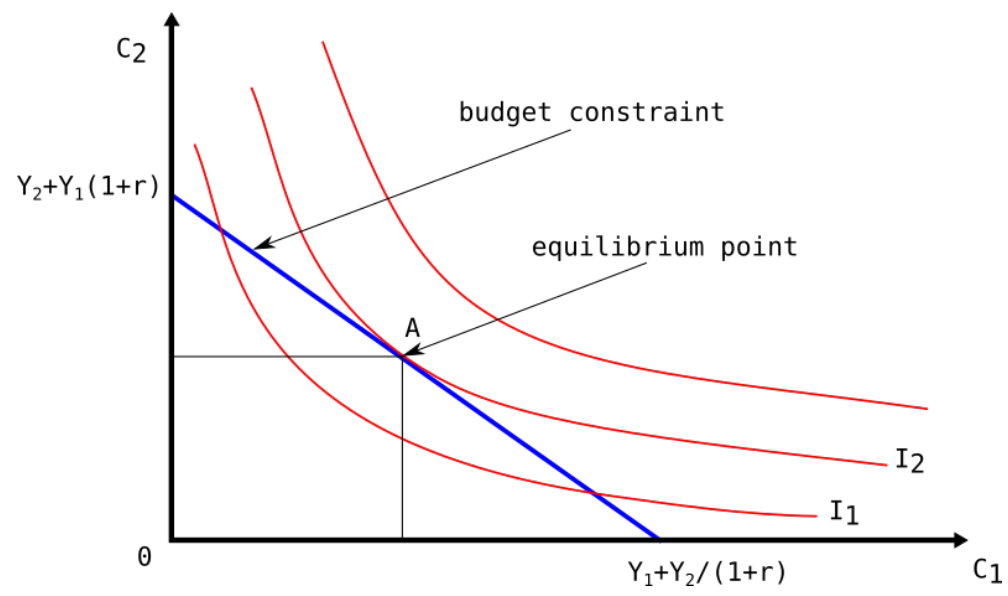

Figure 1. Intertemporal choice exerted by the consumer

curves[19] $\left(I_{1}, I_{2} \ldots I_{n}\right)$ can be part of an indifference map and illustrate different bundles of goods between which a consumer is indifferent. There is an infinity of indifference curves in microeconomic theory and in this case they represent different utility levels. The slope of the indifference curve is the marginal rate of substitution ${ }^{1}$ of goods consumption in different time periods (how many extra goods would you need to consume in $t_{2}$ to give the same level of utility if you consumed one in $t_{1}$ ).

An interesting concept of this model is that we can consume now money that we make in the future by borrowing that amount. The graph is a model of saving and consumption with the interest rate of $r$ over time periods $\left(C_{1}, C_{2}\right.$ represent consumption over periods of time and $Y_{t}$ represents income for time $t)$. The consumer will have to maximize utility $U\left(C_{1}, C_{2}\right)$ but under the constraint represented below:

$$
C_{1}+C_{2} /(1+r)=Y_{1}+Y_{2} /(1+r)
$$

Human behavior is inherently difficult to model, due to the dynamic interactions that can be observed between agents. However, experimental data shows that there are (unwritten) social rules that can be used to model human reactions in some environments[1].

In this paper I will discuss the existing discount functions and our new proposed hybrid models. We conducted our own experiment for sample data

\footnotetext{
${ }^{1}$ The marginal rate of substitution $(M R S)$ represents the rate at which economic agents will substitute one good for another while maintaining the same utility .
} 
and tested the proposed functions. The findings showed better results and a good contribution to our multidimensional economic agent.

The remainder of the article is structured as follows. Section 2 is focused on the concept of temporal discounting and other related papers. Section 3 will cover current experiments from the scientific literature and the zeroshot capability of GPT-3 regarding time preference. Section 4 describes our proposed functions and the methodology we employed, then in Section 5 we will examine the results. Section 6 presents our conclusions and directions for future research.

\section{BACKGROUND}

2.1. Temporal discounting. Temporal discounting (delay discounting, time preference) refers to the observed phenomenon where agents value the same good differently based on the time of consumption. Certainly, a good can be any product that is desired and provides utility ${ }^{2}$ to a consumer.

There are many models that represent temporal discounting or time preference. The models are simplified mathematical versions of discounting in a complex system and are used to explain, analyze and predict behavior and interactions of economic agents. Several differences between the models exist, specifically between the discount functions and we will present some of the most used variations.

The model we presented earlier assumes that the consumers make choices by discounting the present value of their consumption and income exponentially into the future, using the same interest rate. In other words, in ideal markets, both firms and individuals borrow or lend until their marginal rate of substitution between consumption today and consumption tomorrow equals the interest rate $[25,12]$.

Before continuing to mathematical formulae of the function, we should explain several important economic terms for those without an economic background. The discount factor, let's call it $\delta$, is the amount a future value must be multiplied with in order to get the present value and can be defined with the formula

$$
\delta=\frac{1}{1+\rho}
$$

We can extract the discount rate as

\footnotetext{
${ }^{2}$ In economics, utility is a measure of the total satisfaction received from consuming a good or a service. It was introduced by Daniel Bernoulli in 1738
} 


$$
\rho=\frac{1-\delta}{\delta}
$$

The discount rate $\rho$ refers to the interest rate used in discounting. More intuitive examples can be found in the fourth chapter of Behavioral Economics by Edward Cartwright (2011) [7] or in the scientific literature in general. A more general equation for future cash flows is:

$$
P V=F V * \frac{1}{(1+r)^{n}}
$$

where PV $=$ Present Value, FV $=$ Future Value, Discount Factor $=\frac{1}{(1+r)^{n}}$, $\mathrm{n}=$ time and $\mathrm{r}=$ discount rate

2.2. Exponential discounting. Exponential discounting is a very simple way to model choice over time and is by far the most common way used in economics, because of it's simplicity. The standard economic model of exponential discounting was proposed by Samuelson in 1937[23] and the general formula is:

$$
f_{E}(D)=e^{-k D}
$$

where $f(D)$ is the discount factor, $D$ is the delay and $\mathrm{k}$ is a parameter which determines the rate at which value decreases with the time delay. A larger $\mathrm{k}$ can be associated with a steeper discounting of the value of a future reward[11].

We can define total utility in this case by:

$$
u_{T}=\sum_{t=1}^{T} \delta^{t-1} u_{t}
$$

where $u_{t}$ is the utility in time $t$, and $\delta$ is the exponential discount factor. We can rewrite that in continuous time as:

$$
u^{T}=\int_{0}^{T} e^{-\rho t} u_{t}
$$

In Figure 2a we plotted the exponential discount function for different values of $\delta$. The top line is the theoretical limit where no discounting occurs.

There are several anomalies in this model[17], recognized even by Samuelson[23] when he proposed the DU model. He stressed that "it is completely arbitrary to assume that the individual behaves so as to maximize an integral of the form envisaged in (7)"

One of the most prominent anomalies is the constant rate of discount assumption. Empirical evidence suggests that discount rates fall over time. We 
can prove the constant rate by calculating the change in discounting over two consecutive periods of time:

$$
\frac{D(t)}{D(t-1)}=\frac{\delta^{t}}{\delta^{t-1}}=\delta
$$

We can observe the constant factors in Figure 2b.

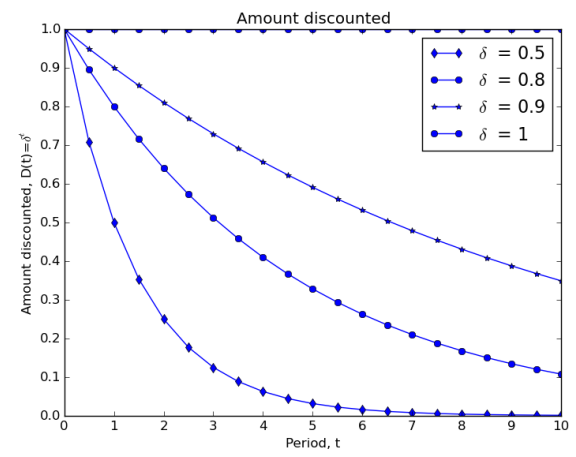

(A) Exponential discount function $\delta^{t}$ versus t

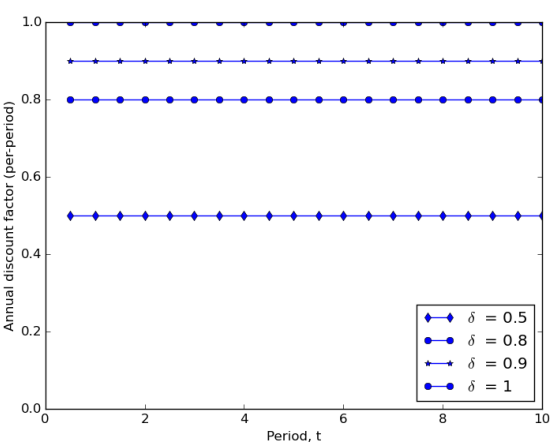

(B) Annual discount factor (per-period), $e^{\ln \left(f_{E}(t)\right) / t}$

Figure 2. Temporal discounting

2.3. Hyperbolic discounting. With the exponential discount function we assumed a constant discount factor $\delta$ and "discount rate" [21] $\rho$ so that:

$$
\delta_{t}=\delta^{t}=\left(\frac{1}{1+\rho}\right)^{t}
$$

Much of the empirical data from both humans and animals contradicts the predictions of exponential discounting [10]. An alternative notion of hyperbolic discounting was developed by psychologists (Ainslie, 1975; Chung \& Herrnstein, 1967; Herrnstein, 1981; Rachlin, 1970), and Mazur (1987) formalized the current standard hyperbolic model [24]:

$$
V=\frac{A}{1+k t}
$$

where $\mathrm{k}$ is a discounting parameter that scales the degree of preference for immediate rewards. Hyperbolic discounting corresponds to simple interest[22]. 
Hyperbolic discounting also permits time-inconsistency. For example you may agree to wait an year and a month for a larger cash prize instead of taking a smaller prize after an year, but you might change your mind after an year and take the money. The Mazur hyperbolic discounting model was initially developed to describe pigeon data and tends to "overpredict subjective value at shorter delays, while underpredicting it at longer delays" [16].

Several researchers have modified his model by adding more parameters to better fit the data. Rachlin (2006) added an exponent $\sigma$ to the time delay, which allows a more flexible relationship between value and delay:

$$
V=\frac{A}{1+k t^{\sigma}}
$$

2.4. Quasi-Hyperbolic discounting. The "quasi-hyperbolic" discount function, proposed by Laibson (1997) [14] as the "Golden Eggs" model assumes that demand follows profits, and it illustrates why consumers have assetspecific marginal consumption propensities. According to the model, financial creativity may be to blame for the continuing downturn in US savings rates.

$$
f_{Q H}(D)=\beta \times \delta^{D}
$$

\section{EXPERIMENTS AND RELATED WORK}

3.1. Thaler experiment. Thaler [25] conducted a study where he asked respondents to state an amount that would be equivalent to receiving $\$ 15$ now. The time periods were one month, one year and ten years. The average response was $\$ 20$ for one month, $\$ 50$ for one year and $\$ 100$ for 10 years ( We can calculate the corresponding annual discount rates (R): $345 \%, 120 \%$ and 19\%)[10]. The same pattern was found by Uri Benzion, Amnon Rapoport, and Joseph Yagil (1989), Gretchen B. Chapman (1996), Chapman and Arthur S. Elstein (1995), John L. Pender (1996), Daniel N. Heller (1993), Stevens, Jeffrey R. (2016).

We used:

$$
R=\frac{\ln \left(\frac{F V}{P V}\right)}{t}[* 100]
$$

where $\mathrm{R}$ is the annual discount rate in percentage, $\mathrm{FV}$ is the future value, $\mathrm{PV}$ is the present value and $t$ is the time period.

The rates were calculated using compound interest with continuous compounding: $F V=P V * e^{R t}$, where $\mathrm{R}$ is the decimal equivalent of the rate of interest expressed as a percentage and $t$ represents time. 
The $e$ constant was discovered by Jacob Bernoulli in 1683 by studying compound interest. The problem was as follows: "An account starts with $\$ 1$ and pays 100 percent interest per year. If the interest is credited once, at the end of the year, the value of the account at year-end will be $\$ 2$. What happens if the interest is computed and credited more frequently during the year?"

Bernoulli noticed that with continuous compounding the account value will reach $\$ 2.7182818 \ldots$.

3.2. Benzion et al. experiment. Another anomaly is the magnitude of payoff effect. The model implicitly assumes that an individual's rate of discount is independent of the size or magnitude of a payoff. Once again, the empirical studies suggests that individuals discount less when faced with larger payoffs. Benzion, Rapoport and Yagil (1989) examined this aspect by varying the amount of money $(\$ 40, \$ 200, \$ 1000, \$ 5000)$ and the time periods $(0.5,12$ and 4 years). They found in all scenarios that the discount rates decrease as the amount of money increases $(0.228,0.18,0.16,0.123$ for a two year period). In Figure 3, the inferred discount rate is :

$$
R=(F / P)^{1 / t}-1
$$

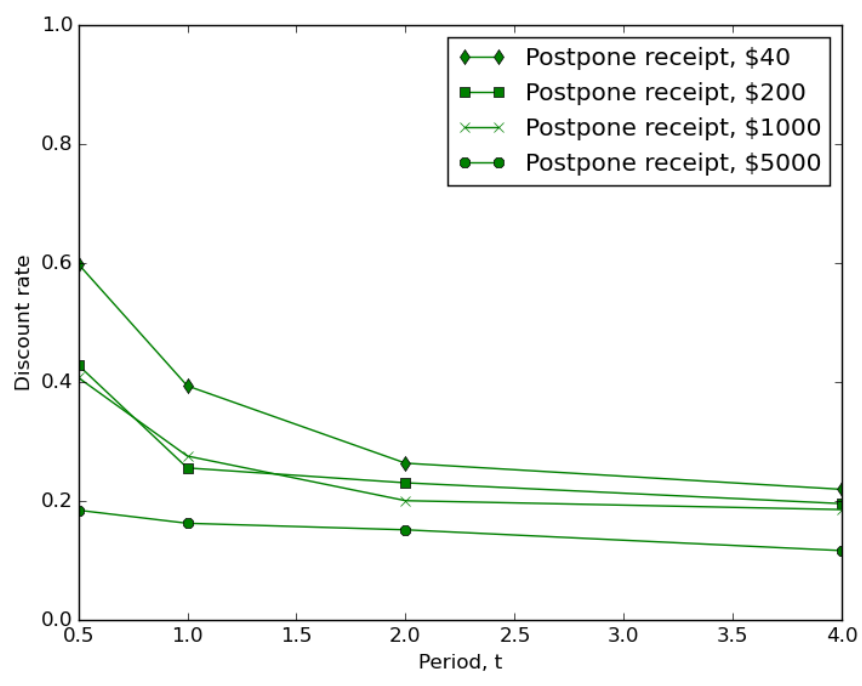

Figure 3. Discount rate - Benzion et al.

In Figure 3 we plot the results of scenario A from Benzion et al. [2] (postpone a receipt), where a person has just earned $\$ y$ for his or her work financially solid public institute. Instead of receiving the money, the person is told 
that there is a temporary shortage of funds and is assured payment of another amount of $\$ y$ over t times periods from now.

If we take the raw average responses and calculate the discount factor $(D F=P V / F V)$, we can plot the results from Figure 4a. With that we compute the annual discount factor from Figure 4b [7]. Figure 4b shows that there is a short-term impatience (The discount factor is higher the longer they had to wait) and an absolute magnitude effect (the larger the sum of money, the larger the estimated discount factor).

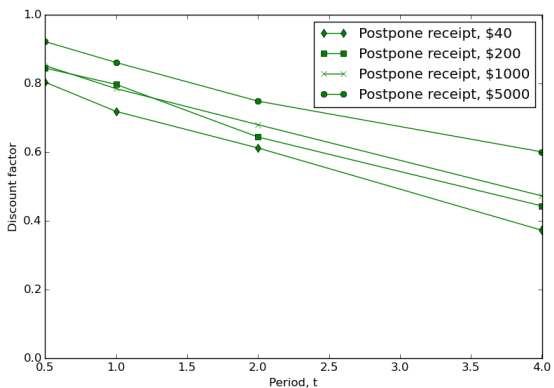

(A) Amount discounted

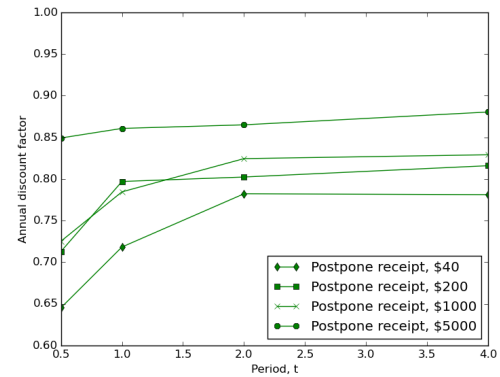

(B) Annual discount factor

Figure 4. Benzion et al. experiment

Next find the best parameter $\delta$ from $D(t)=\delta^{t}$ that fits the experimental data from Figure 4a. We plot the resulting exponential function in Figure 5.

3.3. Benzion and Yagil experiment. This experiment[3] conducted by Benzion and Yagil reexamined the behavior of subjective discount rates across several dimensions: financial scenario, time delay and the monetary sum of the cash flow. They used subsamples of 105 subjects from undergraduates, graduates and higher academic degree. The emerging pattern is similar with other experimental data from literature: the discount rates are decreasing with the time delay and the sum of the cash flow, and are higher for a postpone-a-receipt scenario than for a postpone-a-payment scenario. We can observe their results in Figure 6a, where the mean discount rates are plotted over time t. In their survey they used a scenario A (postponing a receipt), scenario B(postponing a payment), 3 time periods (0.5, 2,5 years) and 3 sum variations $(\$ 200, \$ 600$, $\$ 5000)$

Based on (14) we can determine the future discounted value:

$$
F=P(1+R)^{T}
$$




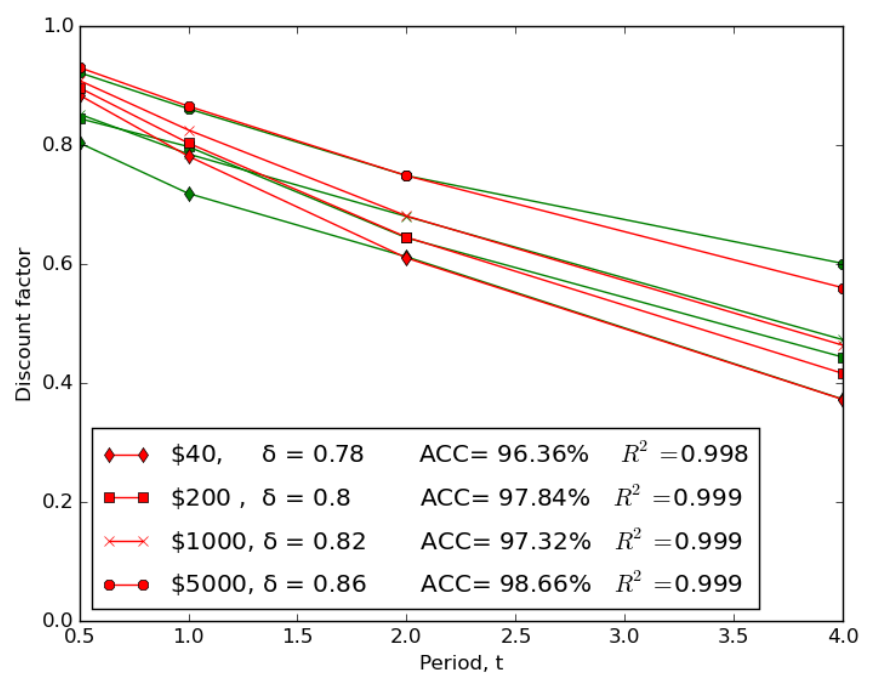

Figure 5. Fitted exponential function - Benzion et al.

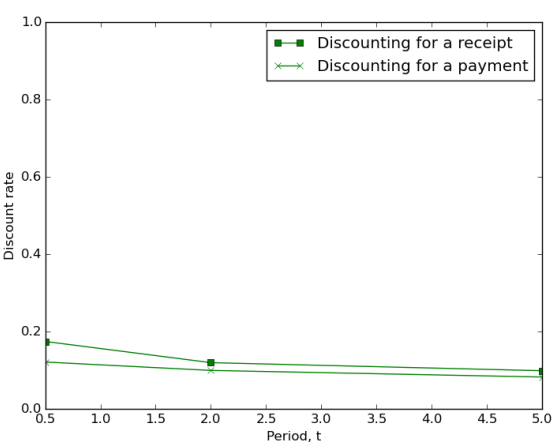

(A) Amount discounted

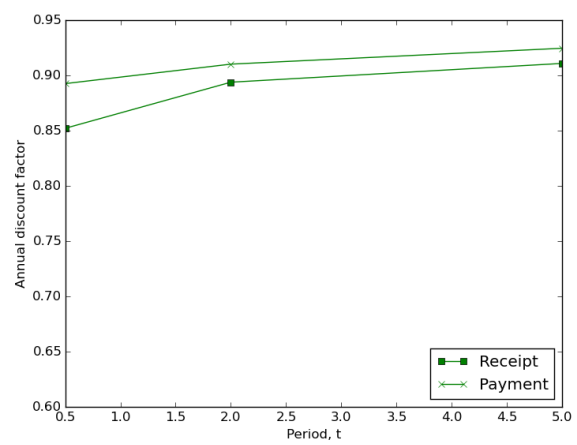

(B) Annual discount factor

Figure 6. Uri Benzion and Joseph Yagil Experiment

where $F$ is the future value of a cashflow, $P$ is the present value, $R$ represents the discount rate and $T$ the time period.

3.4. A general model of temporal discounting. Wouter and Samuel [4] argued that certain behaviours like impulsivity are inexplicable with classic 
models. As a result, psychological models of temporal discounting have now effectively replaced classical economic theory.

This is consistent with our own results in Section 5 and represents a positive context for our own proposed solution in Section 4.2.

In [4] they presented a brain-based discounting model that overcomes some constraints, while retaining much of the practical structure of the hyperbolic discount equation.

They used neuroscience-based theory to create a new model that accounts for several well-known contextual effects, with a simplified discount function seen in (16).

$$
D_{\tau}=\omega \delta_{1}^{\tau}+(1-\omega) \delta_{2}^{\tau}
$$

where $\omega$ indicates the relative involvement of each system in a given decision (McClure, Ericson et al.) [15]

3.5. GPT-3 Experiment. Generative Pre-trainer Transformer 3 (GPT-3) is a language model developed by OpenAI[6], an artificial intelligence research and deployment organization. The model uses historical values to forecast future data (autoregressive) and is based on feed-forward neural networks. GPT-3 is trained with 175 billion parameters, making it a state-of-the-art language model and the largest one at its launch, in 2020. The previous largest model was Microsoft's Turing NLG, with 17 billion parameters, 10 times smaller than GPT-3[20].

Their scaled up approach significantly enhanced task-agnostic, few-shot efficiency[6], competing with other state-of-the-art fine-tuning models[18].

Although this model is usually employed in NLP use cases, the generative attribute with the options of zero-shot and few-shot learning make it a good candidate for our experiment. I could not find any examples of such studies in the literature being conducted so far.

We tested the standard GPT-3 model in making temporal choices by using the OpenAI Playground and the Q\&A preset with temperature set initially to 0 . Temperature is a parameter for stochastic values and controls the randomness of the response. A value of 0 causes the engine to be deterministic, which means it will always produce the same output for a given input text, and a value of 1 causes the engine to take the most chances and use the most imagination.

There are variations in this strategy and we noticed them right away. We were able to communicate with the model using written English and receive written answers as responses, equivalent to our human survey. 
We used adapted questions from our survey, to make things easier for the model. For example:

"Would you prefer $\$ 50$ now or $\$ 500$ in a year?"

The answers were formulated like this:

"I would prefer $\$ 50$ now."

TABLE 1. GPT-3 Q\&A answers for temporal choices

\begin{tabular}{llrc}
\hline Value now $(\$)$ & In a year $(\$)$ & Answer & Temperature \\
\hline 50 & 500 & "I would prefer \$ 50 now." & 0.0 \\
50 & 5000 & "I would prefer \$ 50 now." & 0.0 \\
50 & 50000 & "I would prefer \$ 50 now." & 0.0 \\
\hline
\end{tabular}

We experimented with the model's temperature parameter and quickly noticed that the zero-shot version (no added training) always prefers the offered non-zero value in the present, regardless of the future amount.

By training the model with several examples we are able to simulate more human-like responses and this will be the main research for a future study in our MEA project.

\section{Methodology}

In this section, we will discuss the approach we used to validate existing models as well as the experiments we conducted to demonstrate the accuracy of our proposed hybrid model.

We find an optimum $\delta$ for the exponential function and plot the results in Figure 7. In the following sections we will also use this data to measure predictive accuracy between the models and the experimental data.

4.1. The dataset. After our study of scientific literature, we were concerned that most of the articles and experiments offered interpreted data with average discount rates and average responses. We wanted sample raw data with original answers to get a better understanding of the topic.

Therefore we conducted a survey with students from Babes-Bolyai University of Cluj-Napoca and other participants from an on-line community (reddit). There were a total of 118 responses, with 52 respondents being computer science undergraduate students and 66 online respondents from Europe and USA. We used Google Forms as a survey tool, to gather the responses.

By using the template: 


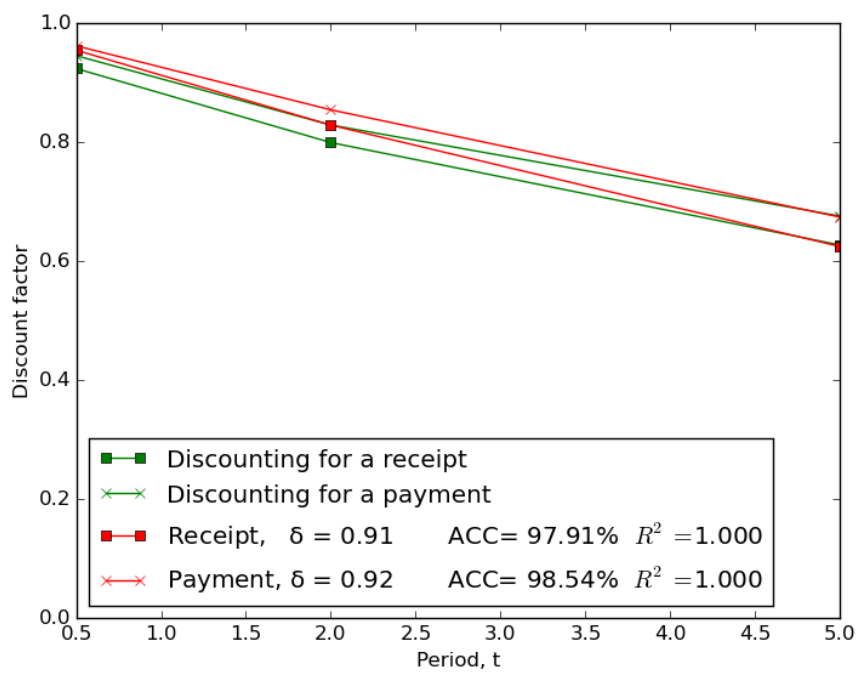

Figure 7. Fitted exponential function - Uri Benzion and Joseph Yagil, 2002

TABLE 2. Sample survey answers

\begin{tabular}{lllll|rrrrr}
\hline & \multicolumn{10}{c}{$\mathbf{5 0} \$$} & $\mathbf{5 0 0 0} \$$ \\
\hline $3 \mathrm{mo}$ & $6 \mathrm{mo}$ & $1 \mathrm{yr}$ & $2 \mathrm{yr}$ & $4 \mathrm{yr}$ & $3 \mathrm{mo}$ & $6 \mathrm{mo}$ & $1 \mathrm{yr}$ & $2 \mathrm{yr}$ & $4 \mathrm{yr}$ \\
\hline 200 & 400 & 600 & 1000 & 2000 & 8000 & 10000 & 15000 & 20000 & 35000 \\
75 & 100 & 300 & 500 & 1000 & 5999 & 7420 & 8499 & 9999 & 14000 \\
60 & 75 & 100 & 200 & 400 & 6000 & 7500 & 8000 & 15000 & 25000 \\
65 & 80 & 100 & 150 & 300 & 6000 & 6666 & 8000 & 10000 & 12500 \\
100 & 300 & 1000 & 2000 & 5000 & 10000 & 10000 & 12000 & 15000 & 20000 \\
75 & 100 & 300 & 500 & 1000 & 5200 & 5500 & 6000 & 7000 & 10000 \\
52 & 54 & 56 & 60 & 70 & 5200 & 5500 & 6000 & 7000 & 8000 \\
300 & 500 & 700 & 1000 & 2000 & 6000 & 8000 & 10000 & 15000 & 20000 \\
55 & 60 & 80 & 130 & 300 & 5100 & 5500 & 6000 & 7000 & 8000 \\
\hline
\end{tabular}

You are indifferent to $Y \$$ now vs $X \$$ in $t$ years. Write the $X$ amount below 
we questioned the subjects to state indifference for receiving money over 6 months, 1 year, 2 years and 4 years periods. The money amount varied $(\$ 50$ and $\$ 5000)$. We can observe a sample set with the answers in Table 2 below.

We specifically asked the following question:

"You have a bank savings plan which just achieved maturity and the bank manger offers you the choice to invest again in another similar savings plan but with a different maturity time. What amount of money would make you COMPLETELY indifferent about receiving the relevant sum today or receiving a larger sum in the future?"

Based on the results in Table 2 we calculated the discount factors, which can be seen in Figure 8.

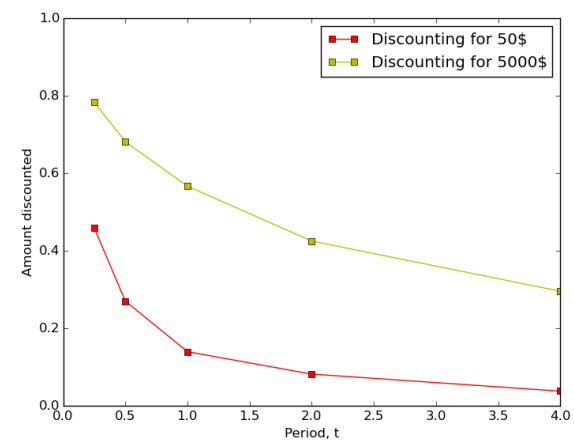

(A) Amount discounted

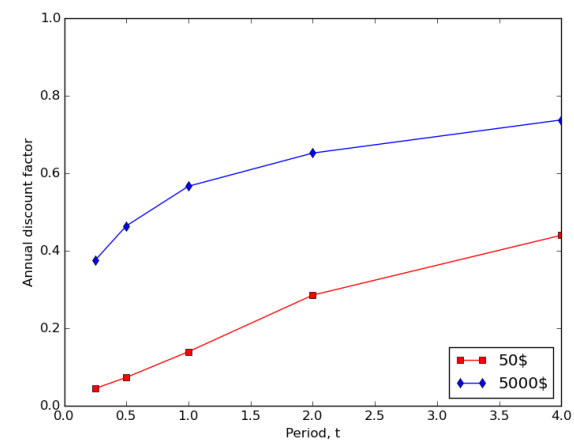

(в) Annual discount factor

Figure 8. Discounted values in our experiment

As compared to older experimental evidence discussed in this paper, the findings show some variations, but they are are consistent with more recent research [8].

4.2. Proposed functions. In this context, we suggest two new functions that can be thought of as hybrids of existing, simplified models. We believe that by using multiple parameters, they can conform better to modern human behaviour, particularly given the anomalies we discovered in the literature.

Equation (17) describes Hybrid Exponential-Hyperbolic discount function:

$$
h y b_{E H}(x)=\frac{\delta^{x}}{1+(\alpha \times x)}
$$


and equation (18) describes the Hybrid Quasi-Exponential-Hyperbolic discount function.

$$
h y b_{Q E H}(x)=\beta \times \frac{\delta^{x}}{1+(\alpha \times x)}
$$

In these functions, $\delta$ is the discount factor and $\alpha$ represents the discounting parameter that scales the degree of desire for instant gratification, with $\beta$ being used to capture present-time bias. In our experiments, the parameters are estimated using the ordinary least squares (OLS) method.

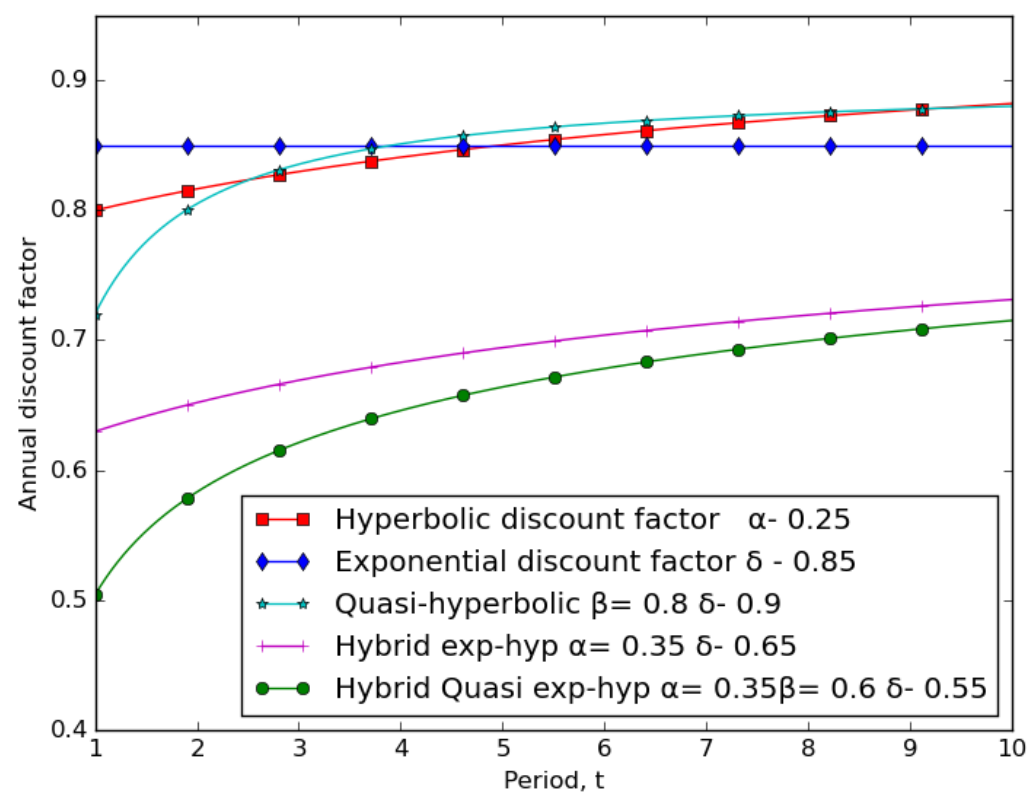

Figure 9. Annual discount factor, function comparison

Figure 11 shows a comparison of current approaches and our suggested hybrid functions for the annual discount factor.

We can observe similarities between the quasi-exponential-hyperbolic function and quasi-hyperbolic, and between exponential-hyperbolic with the hyperbolic discount function. As shown in the following section, the variations are important in our analysis. 


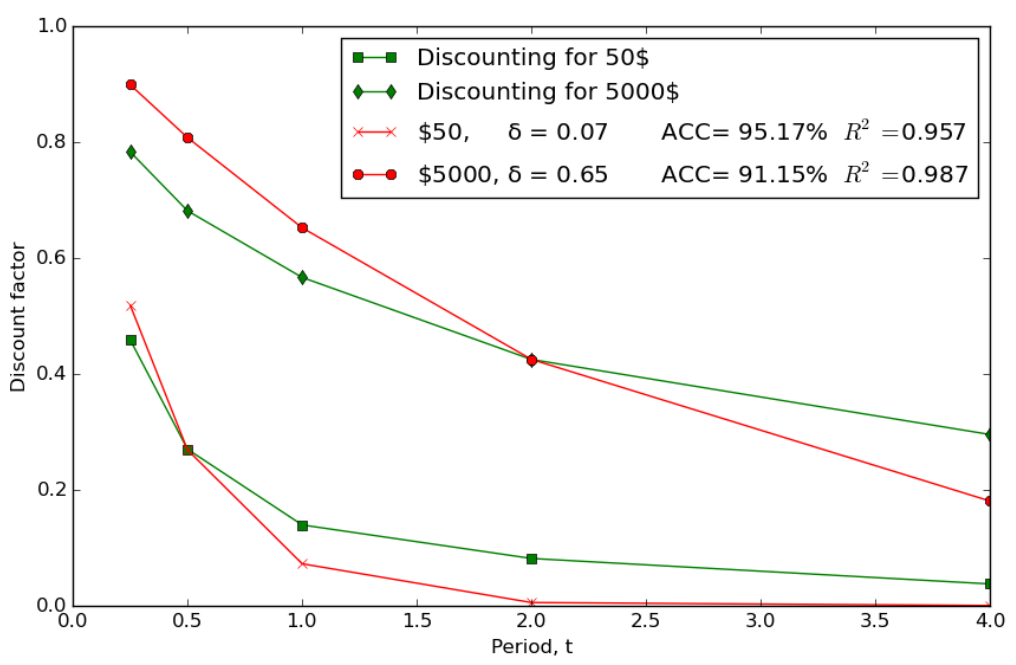

FiguRE 10. Fitted exponential function, using survey data

\section{Results}

We can observe the discount factors in Figure 10, where we fitted the exponential discount function to our data. The outcome shows a $\mathbf{9 5 . 1 7 \%}$ accuracy for the $50 \$$ discount and $\mathbf{9 1 . 1 5 \%}$ for the $5000 \$$ questions.

This suggests that the exponential discount function doesn't fit well on our experimental values, relative to the experiments of Benzion et al. in Figure 5. We believe this is due to the participants' lack of economic awareness or to inconsistencies in the model compared to our results. It also provides us with an ideal setting for testing the models in a real-world situation where data is sparse or uncertain.

A sample of the answers from our survey can be observed in Table 2. We tested all multiple functions on our survey data and the results can be observed in Table 3.

The visual representation can be seen in Figure 11, where our hybrid functions performed very well, with 99.39 \% accuracy for Quasi-ExponentialHyperbolic and $\mathbf{9 8 . 6 5 \%}$ accuracy for Exponential-Hyperbolic.

The standard model of exponential discounting did the worst, with $\mathbf{8 9 . 0 2}$ \%, which is consistent with other examples in the recent literature [4] [8]. 


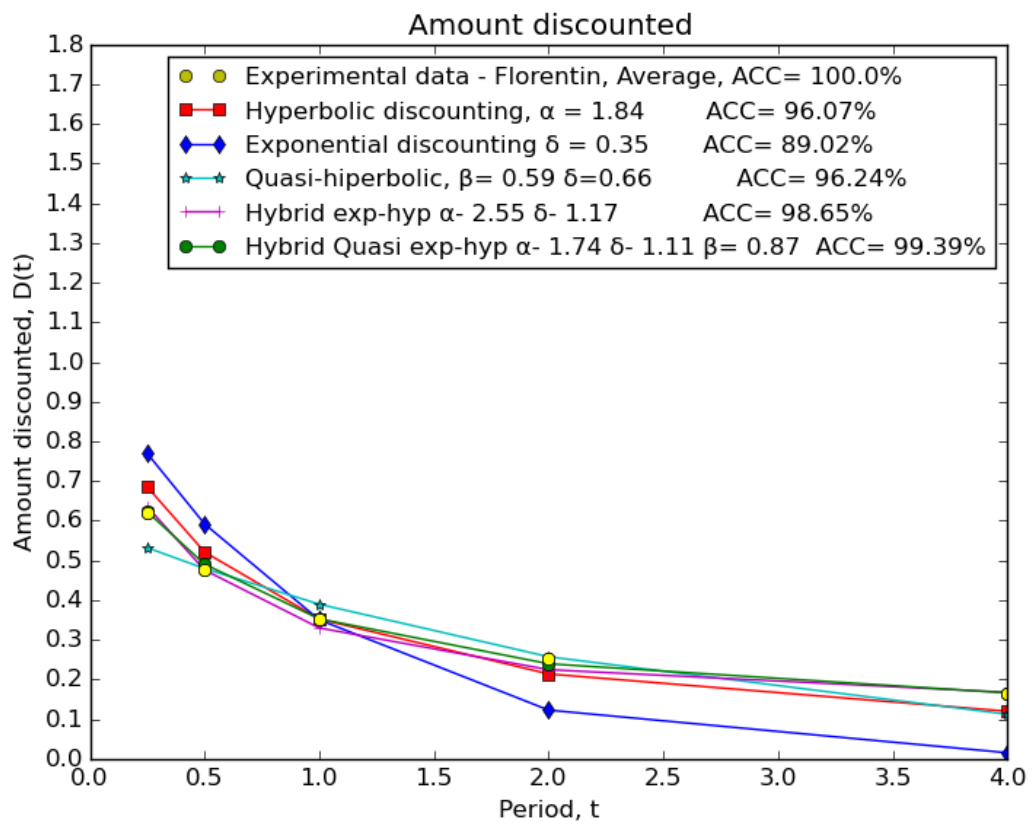

Figure 11. Accuracy on real data (average)

TABLE 3. Accuracy results for our experimental data

\begin{tabular}{lr}
\hline Discount function & ACC \\
\hline Hybrid Quasi-Exponential-Hyperbolic & $99.39 \%$ \\
Hybrid Exponential-Hyperbolic & $98.65 \%$ \\
Quasi-Hyperbolic & $96.24 \%$ \\
Hyperbolic & $96.07 \%$ \\
Exponential & $89.02 \%$ \\
\hline
\end{tabular}

\section{CONClusions AND FURTher WORK}

In this article, we provided an analysis of the temporal discounting phenomena found in economic processes. Using studies from the literature, we evaluated existing discount functions and proposed new hybrid solutions based on our own experimental data. 
Our proposed functions performed well and we determined very good results for the Quasi-Exponential-Hyperbolic function, with up to $\mathbf{9 9 . 3 9 \%}$ accuracy.

According to our findings, the standard functions performed well on older data sets and struggled to model behavior on newer data, while our proposed solutions show a very good potential to model consumers constraining their own future choices.

We also obtained intriguing results with zero-shot and GPT-3 learning in the form of inter-temporal preference (always accepting the present value), yet further investigation is required.

This paper represents an important phase in our MEA[5] research, in which we developed a theoretical model that can be used to simulate and forecast human actions in complex scenarios.

Further work will include a more in-depth analysis on few-shot learning models such as GPT-3 and their ability to simulate inter-temporal choices.

\section{REFERENCES}

[1] Alahi, A., Ramanathan, V., Goel, K., Robicquet, A., Sadeghian, A.A., Fei-Fei, L., Savarese, S.: Chapter 9 - learning to predict human behavior in crowded scenes. In: Murino, V., Cristani, M., Shah, S., Savarese, S. (eds.) Group and Crowd Behavior for Computer Vision, pp. 183-207. Academic Press (2017)

[2] Benzion, U., Rapoport, A., Yagil, J.: Discount rates inferred from decisions: An experimental study. Management science 35(3), 270-284 (1989)

[3] Benzion, U., Yagil, J.: Decisions in financial economics: An experimental study of discount rates. Advances in Financial Economics 7, 19-40 (2002)

[4] Van den Bos, W., McClure, S.M.: Towards a general model of temporal discounting. Journal of the experimental analysis of behavior 99(1), 58-73 (2013)

[5] Bota, F., Simian, D.: Embedding human behavior using multidimensional economic agents. In: Simian, D., Stoica, L.F. (eds.) Modelling and Development of Intelligent Systems. pp. 3-19. Springer International Publishing, Cham (2021)

[6] Brown, T.B., Mann, B., Ryder, N., Subbiah, M., Kaplan, J., Dhariwal, P., Neelakantan, A., Shyam, P., Sastry, G., Askell, A., et al.: Language models are few-shot learners. arXiv preprint arXiv:2005.14165 (2020)

[7] Cartwright, E.: Behavioral economics, vol. 22. Routledge (2014)

[8] Esopo, K., Mellow, D., Thomas, C., Uckat, H., Abraham, J., Jain, P., Jang, C., Otis, N., Riis-Vestergaard, M., Starcev, A., et al.: Measuring self-efficacy, executive function, and temporal discounting in kenya. Behaviour Research and Therapy 101, 30-45 (2018)

[9] Fisher, I.: The theory of interest. New York 43 (1930)

[10] Frederick, S., Loewenstein, G., O'donoghue, T.: Time discounting and time preference: A critical review. Journal of economic literature 40(2), 351-401 (2002)

[11] Green, L., Myerson, J.: Exponential versus hyperbolic discounting of delayed outcomes: Risk and waiting time. American Zoologist 36(4), 496-505 (1996)

[12] Keller, L.R., Strazzera, E.: Examining predictive accuracy among discounting models. Journal of Risk and Uncertainty 24(2), 143-160 (2002)

[13] Keynes, J.M.: General theory of employment, interest and money. Atlantic Publishers \& Dist (2007) 
[14] Laibson, D.: Golden eggs and hyperbolic discounting. The Quarterly Journal of Economics 112(2), 443-478 (1997)

[15] McClure, S.M., Ericson, K.M., Laibson, D.I., Loewenstein, G., Cohen, J.D.: Time discounting for primary rewards. Journal of neuroscience 27(21), 5796-5804 (2007)

[16] McKerchar, T.L., Green, L., Myerson, J., Pickford, T.S., Hill, J.C., Stout, S.C.: A comparison of four models of delay discounting in humans. Behavioural processes 81(2), 256-259 (2009)

[17] Musau, A.: Modeling alternatives to exponential discounting (2009)

[18] Nagabandi, A., Kahn, G., Fearing, R.S., Levine, S.: Neural network dynamics for model-based deep reinforcement learning with model-free fine-tuning. In: 2018 IEEE International Conference on Robotics and Automation (ICRA). pp. 7559-7566. IEEE (2018)

[19] Pareto, V.: Manuale di economia politica con una introduzione alla scienza sociale (manual of political economy). Milano: Societa Editrice Libraria (1919)

[20] Rajbhandari, S., Rasley, J., Ruwase, O., He, Y.: Zero: Memory optimizations toward training trillion parameter models. In: SC20: International Conference for High Performance Computing, Networking, Storage and Analysis. pp. 1-16. IEEE (2020)

[21] Rasmusen, E., et al.: Some common confusions about hyperbolic discounting. In: Working Paper (2008)

[22] Read, D.: Intertemporal choice. Blackwell handbook of judgment and decision making pp. 424-443 (2004)

[23] Samuelson, P.A.: A note on measurement of utility. The Review of Economic Studies 4(2), 155-161 (1937)

[24] Stevens, J.R.: Intertemporal similarity: Discounting as a last resort. Journal of Behavioral Decision Making 29(1), 12-24 (2016)

[25] Thaler, R.H.: Some empirical evidence on dynamic inconsistency. Quasi rational economics 1, 127-136 (1981)

Department of Computer Science, Faculty of Mathematics and Computer Science, Babeș-Bolyai University, 1 Kogălniceanu St., 400084 Cluj-Napoca, RomaNIA

Email address: florentin.bota@ubbcluj.ro 